EPJ Web of Conferences 43, 05011 (2013)

DOI: $10.1051 /$ epjconf/20134305011

(C) Owned by the authors, published by EDP Sciences, 2013

\title{
The angular momentum of isolated white dwarfs
}

\author{
G. Fontaine ${ }^{1, a}$, P. Brassard ${ }^{1}$ and S. Charpinet ${ }^{2}$ \\ ${ }^{1}$ Département de Physique, Université de Montréal \\ ${ }^{2}$ CNRS, Université de Toulouse, UPS-OMP, IRAP
}

\begin{abstract}
This is a very brief report on an ongoing program aimed at mapping the internal rotation profiles of stars through asteroseismology. Three years ago, we developed and applied successfully a new technique to the pulsating GW Vir white dwarf PG 1159-035, and were able to infer that it rotates very slowly and rigidly over some $99 \%$ of its mass. We applied the same approach to the three other GW Vir pulsators with available rotational splitting data, and found similar results. We discuss the implications of these findings on the question of the angular momentum of white dwarfs resulting from single star evolution.
\end{abstract}

\section{ASTROPHYSICAL CONTEXT}

White dwarf stars represent the final phase of the evolution of some $95-97 \%$ of all stars. If stars were to keep their angular momentum throughout their evolution, their white dwarf descendants, due to their compact nature, would all rotate relatively rapidly, with periods of order of seconds. Observations of their photospheres show, however, that they rotate much slower (at least superficially), with periods (measured mostly through spectroscopy and polarimetry) ranging from a few hours to tens of years. While it is widely believed that most of the angular momentum of an isolated star is lost during important mass loss episodes as it goes through the red giant phases of its evolution, current observations have been unable to reveal how rotation varies with depth in a white dwarf. In particular, one could never exclude the possibility that the internal regions, inaccessible to direct observations, could spin quite rapidly and, hence, "hide" a substantial fraction of the original angular momentum. The reader will have realized here that we are referring to isolated objects that are the products of single star evolution.

Asteroseismic inferences about the rotation state of pulsating white dwarfs have also been made under the assumption of solid body rotation, but nothing could be said about the extent of the stellar zone actually probed by the observed pulsation modes. In other words, those inferences did not reveal what specific region of a pulsating star (e.g., core or envelope) imprints most on the rotational signature left on the pulsation data. Using a novel method that we developed recently [1], we are now able to map or constrain the internal rotation profile of a pulsating white dwarf. Our method can most efficiently be used for GW Vir pulsators, for which the internal rotation profile can be mapped essentially over the full mass, thus inferring or constraining the total angular momentum. After having demonstrated that PG 1159-035 rotates slowly $(33.67 \pm 0.24 \mathrm{~h})$ and rigidly over some $99 \%$ of its mass (it should be noted that this mapping is more extensive than what has been achieved so far in the Sun through helioseismology, and is unique to asteroseismology; see Fig. 1), we have extended our approach to three other GW Vir pulsators. We briefly report on some of our results here.

\footnotetext{
a e-mail: fontaine@astro.umontreal.ca
}

This is an Open Access article distributed under the terms of the Creative Commons Attribution License 2.0, which permits unrestricted use, distribution, and reproduction in any medium, provided the original work is properly cited. 


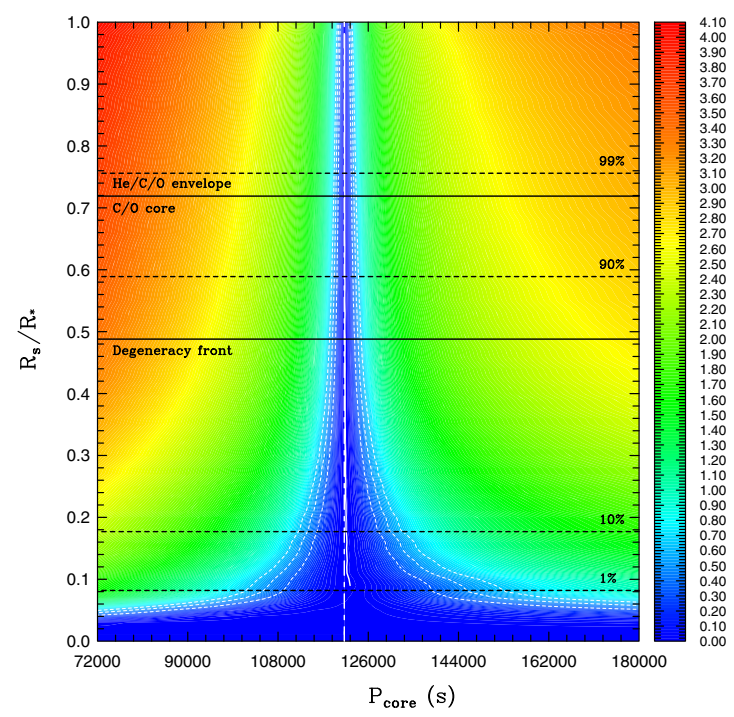

Figure 1. Inferred internal rotation profile of PG $1159-035$.

\section{RESULTS}

Our method consists in analyzing the available rotational data (observed frequency splittings within multiplet structures) under the assumptions that 1) the star rotates slowly, and 2) it rotates as a rigid body. The inferred rotation period, $P_{\text {solid }}$, can be used after the fact to verify if the star indeed rotates slowly, in which case $P_{\text {solid }}$ should be much larger than the periods of the pulsation modes used in the experiment. Next, we have devised a number of ways to test for rigid rotation. Details will be provided elsewhere.

In addition to PG 1159-035, the other three GW Vir pulsators investigated are PG 0122+200 $\left(P_{\text {solid }}=41.80 \pm 2.05 \mathrm{~h}\right), \mathrm{PG} 2131+066\left(P_{\text {solid }}=5.15 \pm 0.18 \mathrm{~h}\right)$, and RXJ $2117+3412\left(P_{\text {solid }}=27.71 \pm\right.$ $1.80 \mathrm{~h})$. We found that all of these stars rotate slowly and rigidly. In particular, we estimate that the ratio of the global rotation energy to the thermal energy varies from $3.2 \times 10^{-8}$ in PG $0122+200$ (our slowest rotator) to $1.6 \times 10^{-6}$ in PG $2131+066$ (our fastest rotator). These numbers prove eloquently that rotation has no longer any significant role to play in the destiny of these stars.

The four GW Vir pulsators investigated in our study are fully representative of the post-AGB evolutionary phases. They therefore indicate that, quite generally, isolated white dwarf stars (that are the products of single star evolution) have lost essentially all of their angular momentum at this stage of their evolution.

There exist two families of competing theories with respect to the mechanisms of angular momentum transport and loss during stellar evolution. It is well established that an isolated star loses angular momentum in the red giant phases of its evolution, during which there are important stellar winds. These winds bring with them essentially all of the angular momentum of the extended outer convective envelope of a red giant, but the question has been whether or not there exists a strong coupling in terms of angular momentum transfer between the convective envelope and the radiative core. Our results show that strong coupling mechanisms must be at work in real stars, and that the latter globally spin very slowly by the time they have reached the end of their life in the form of isolated white dwarfs.

\section{Reference}

[1] Charpinet, S., Fontaine, G., \& Brassard, P., Nature, 461, (2009) 501 